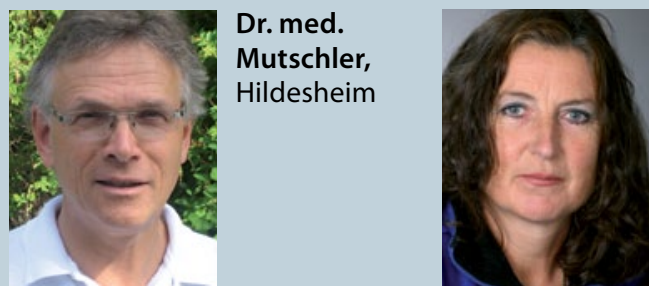

Dr. med.

Kirsten

Stollhoff,

Hamburg

hätten, insgesamt waren $8 \%$ der Befragten betroffen. Im weiteren Verlauf des Erwachsenwerdens war diese Zahl stark rückläufig. Von den erwähnten $8 \%$ hatten die meisten das Verhalten aufgegeben, sodass nur noch $1 \%$ der jungen Erwachsenen betroffen war. In der Adoleszenz war die Selbstbeschädigung unabhängig assoziiert mit Symptomen von Depression, Angst, antisozialem Verhalten, Alkohol- und Cannabismissbrauch.
Dabei waren die Symptome von Depression und Angst prädiktiv für das Fortbestehen von Selbstbeschädigung im Erwachsenenalter. Die häufigste Form der Selbstbeschädigung bestand im Beifügen von Hautverletzungen oder Verbrennungen.

Moran P et al. The natural history of self-harm from adolescence to young adulthood: a populationbased cohort study. Lancet 2012; 379: 236-43
Kommentar: Die gute Botschaft dieser Studie besteht in der überraschend hohen Selbstheilungstendenz von $87 \%$.Zu berücksichtigen ist, dass diese hohe Zahl zustande kommt durch die Befragung der Betroffenen, die größtenteils keine ärztliche Hilfe gesucht haben. Für den Kinderarzt wichtig ist, das gleichzeitige Bestehen von Selbstbeschädigung mit Symptomen von Depression zu registrieren. Hier ist größte Aufmerksamkeit geboten, da Suizidgefahr besteht.

Dr. Hartmut Koch

\title{
Neugeborene durch Nabelschnurdesinfektion schützen
}

\section{Die Desinfektion der Nabelschnur bei Neugeborenen mit Chlorhexidin kann Leben retten. In einer Studie an rund 30.000 Neugeborenen aus Bangladesch konnte dies eindrucksvoll gezeigt werden.}

n Ländern mit hoher Säuglingssterblichkeit können bis zu 50\% der neonatalen Todesfälle auf Infektionen zurückgeführt werden. Viele dieser Infektionen wiederum erfolgen über den Nabelschnurstumpf. Das Ziel einer Studie war es, die Wirksamkeit zweier Vorgehensweisen zur Nabelschnurdesinfektion - einmalige Desinfektion bzw. Desinfektion über sieben Tage - im Vergleich mit einer trockenen Nabelpflege zu beurteilen. Die Untersuchung wurde in Bangladesch von Juni 2007 bis September 2009 bei knapp 30.000 Neugeborenen durchgeführt.

Die Neugeborenensterblichkeit (innerhalb von 28 Tagen nach Geburt) war in der Gruppe mit Einzeldesinfektion (22,5 pro 1.000 Lebendgeburten) niedriger als bei der Gruppe mit trockener Nabelpflege (28,3 pro 1.000 Lebendgeburten; relatives Risiko [RR]: 0,80). Erstaunlicherweise war sie in der Gruppe mit Mehrfachdesinfektion (26,6 pro 1.000 Lebendgeburten) nicht statistisch signifikant niedriger als in der Gruppe mit trockener Nabelschnurpflege (RR: 0,94). Jedoch konnte eine Reduktion bei schweren $\mathrm{Na}$ belschnurinfektionen (d.h. Rötung mit Eiter) bei der Gruppe mit Mehrfachdesinfektion (Risiko pro 1.000 Lebendgeburten $=4,2$ ) gegenüber der trockenen Pflege (Risiko pro 1.000 Lebendgeburten $=1,2$; RR: 0,35) gezeigt werden.

El Arifeen $S$ et al. The effect of cord cleansing with chlorhexidine on neonatal mortality in rural Bangladesh: a community-based, cluster-randomised trial. Lancet 2012; doi:10.1016/S0140-6736(11) 61848-5

Kommentar: In dieser großen randomisierten und kontrollierten Studie wurde überzeugend dargestellt, wie eine einzige Desinfektion des Nabels mit 4\%igem Chlorhexidin (so früh wie möglich nach Geburt appliziert) die Neugeborenensterblichkeit senken hilft. Möglicherweise sind aber auch die Nebeneffekte der Intervention nicht von der Hand zu weisen: Die Familien wurden kurz nach Ge-

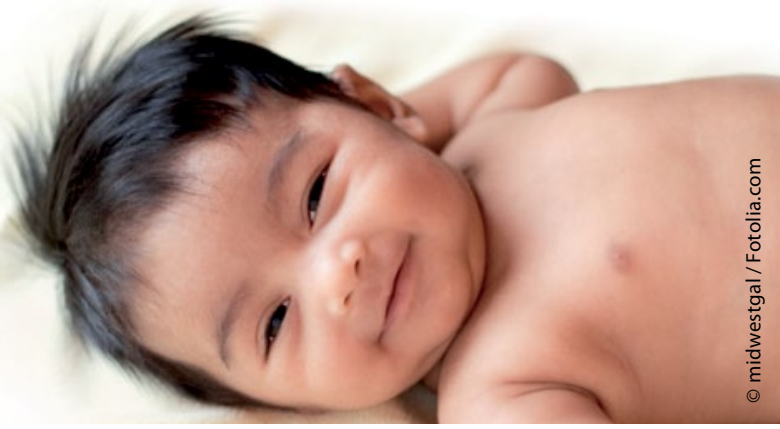

Säuglinge aus Bangladesch überlebten nach einer einmaligen Desinfektions des Nabels signifikant häufiger.

burt von Basisgesundheitshelfern aufgesucht. Die Information über eine wie auch immer geartete Nabelpflege schützt die Neugeborenen vor fragwürdigen lokalen Maßnahmen wie z.B. Asche, Kuhdung oder ähnlichem und fördert ein allgemeines Gesundheitsbewusstsein. Chlorhexidin hat darüber hinaus ein gutes Sicherheitsprofil, ist einfach anzuwenden und preiswert. Es kann somit auch rasch in ein existierendes Basisgesundheitsprogramm eingebaut werden.

Zusammenfassend kann bei Neugeborenen eine Desinfektion der Nabelschnur mit Chlorhexidin lebensrettend sein. Es bedarf jedoch weiterer Untersuchungen, um die optimale Häufigkeit für die Applikation zu ermitteln.

Dr. Ulrich Mutschler 